J. Natn. Sci. Coun. Sri Lanka 1987 15(1): 71-81

\title{
ON ORDINARY LIMITABILITY FACTORS FOR CESARO MEANS
}

\section{YOGACHANDRAN}

Department of Mathematics and Statistics, University of Maiduguri, Nigeria.

(Date of receipt : 23 December 1985)

(Date of acceptance : 24 March 1987)

\begin{abstract}
This paper deals with the problem of finding necessary and sufficient conditions in order that, for some $l^{\prime}, f(x) g(x) \sim l^{\prime} x{ }^{p+q}(C, \mu)$ whenever $f(x) \sim$ $1 \mathrm{x}^{\mathrm{P}}(\mathrm{C}, \lambda)$ for some 1 , where $\mu \geqslant \lambda \geqslant 1, \mathrm{p}>-1, \mathrm{p}+\mathrm{q}>-1$ and $\mathrm{f} \in \mathrm{L}_{\text {loc }}$. This problem is a generalization of a problem considered earlier, ${ }^{*}$ in which $\mu, \lambda$ were replaced by positive integers $r, k, r \geqslant k$ and $l$ and $I^{\prime}$ were zero.
\end{abstract}

\section{Introduction}

Let $f$ be a real function with domain $\leqslant[1, \infty)$. If $f \in \mathrm{L}_{10 \mathrm{c}}$ (i.e. $\mathrm{f}$ is locally Lebesgue integrable) and $\lambda>0$, we define

$$
f_{\lambda}(x)=I_{\lambda} f(x)=\frac{1}{\Gamma(\lambda)} \int_{1}^{x}(x-t)^{\lambda-1} f(t) d t \text {, and set } f_{0}(x)=f(x) .
$$

we say that $f(x)$ is Cesaro limitable of order $\lambda$ in the ordinary sense to 1 , written $\mathrm{f}(\mathrm{x}) \rightarrow 1(\mathrm{C}, \lambda)$ if $\Gamma(\lambda+1) \mathrm{x}^{-\lambda} \mathrm{f}_{\lambda}(\mathrm{x}) \rightarrow 1$ as $\mathrm{x} \rightarrow \infty$. More generally, if $\mathrm{p}>-1 . \mathrm{l} \neq 0$, we write $\mathrm{f}(\mathrm{x}) \sim 1 \mathrm{x}^{\mathrm{p}}(\mathrm{C}, \lambda)$ if

$$
\frac{\Gamma(\lambda+\mathrm{p}+1)}{\Gamma(\mathrm{P}+1)} \mathrm{x}^{-\mathrm{p}-\lambda_{\mathrm{f}}}(\mathrm{x}) \rightarrow 1 \text { as } \mathrm{x} \rightarrow \infty \text {. }
$$

If $\mathrm{p}$ is real, we write $\mathrm{f}(\mathrm{x})=0\left(\mathrm{x}^{\mathrm{p}}\right)(\mathrm{C}, \lambda)\left[\right.$ or $\left.\mathrm{o}\left(\mathrm{x}^{\mathrm{p}}\right)(\mathrm{C}, \lambda)\right]$ if $\mathrm{x}^{-\mathrm{p}-\lambda} \mathrm{f}_{\lambda}(\mathrm{x})=0(1)$ $[$ or $\mathrm{o}(1)]$ as $\mathrm{x} \rightarrow \infty$.

In (5), we found conditions necessary and sufficient in order that $\mathrm{f}(\mathrm{x}) \mathrm{g}(\mathrm{x})=\mathrm{o}\left(\mathrm{x}^{\mathrm{p}+\mathrm{q}}\right)(\mathrm{C}, \mathrm{r})$ whenever $\mathrm{f}(\mathrm{x})=\mathrm{o}\left(\mathrm{x}^{\mathrm{p}}\right)(\mathrm{C}, \mathrm{k})$, where $\mathrm{r}, \mathrm{k}$ are nonnegative integers and $r \geqslant k$. (See (5), Theorem 1). This is the integral analogue of a theorem given in (3). The following theorem is a direct consequence of the above Theorem 1.

THEOREM A : Let $r, k \in / N_{v}, r \geqslant k, p>-1, p+q>-1$. Also let $f \in L_{l o c}$ and $\mathrm{g} \in \mathrm{L}_{\mathrm{loc}}^{\infty}$ if $\mathrm{k}=0, \mathrm{r} \geqslant 1$, and $\mathrm{g}^{\mathrm{k}-1} \in \mathrm{AC}_{\mathrm{loc}}$ if $\equiv \mathrm{k} \geqslant 1$.

Then, conditions necessary and sufficient in order that for some $1^{\prime}$, $\mathrm{f}(\mathrm{x}) \mathrm{g}(\mathrm{x}) \sim \mathrm{1}^{\prime} \mathrm{x}^{\mathrm{p}+\mathrm{q}}(\mathrm{C}, \mathrm{r})$ whenever $\mathrm{f}(\mathrm{x}) \sim 1 \mathrm{x}^{\mathrm{p}}(\mathrm{C}, \mathrm{k})$ for some 1 are : 
(a) (i) $\int_{1}^{\mathrm{x}}|\phi(\mathrm{t})| \mathrm{dt}=0(\mathrm{x})$ as $\mathrm{x} \rightarrow \infty$,

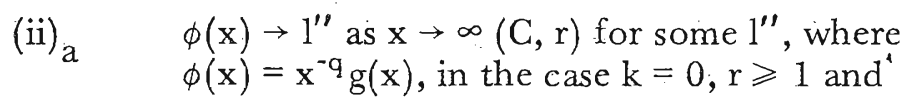

(b) $(\text { i })_{\mathrm{b}} \quad \phi(\mathrm{x})=0(1)$ as $\mathrm{x} \rightarrow \infty$,

(ii) $b_{1} \int^{x} t^{k-q}\left|g^{k}(t)\right| d t=0(x)$ as $x \rightarrow \infty$,

(iii) $_{\mathrm{b}} \quad \phi(\mathrm{x}) \rightarrow \mathrm{l}^{\prime \prime}(\mathrm{C}, \mathrm{r})$ as $\mathrm{x} \rightarrow \infty$,

in the case $r \geqslant k \geqslant 1$.

In this paper, we generalise Theorem A. We replace the integers $r, k$ by real numbers $\mu, \lambda$ respectively, where $\mu \geqslant \lambda \geqslant 1$. We also drop the restriction $\mathrm{g}^{\mathrm{k}-1} \in \mathrm{AC}_{\text {loc }}$. We prove the following theorem.

THEOREM $\mathrm{B}:$ Let $\mu \geqslant \lambda \geqslant 1, \mathrm{p}>-1, \mathrm{p}+\mathrm{q}>-1$ and $\mathrm{f} \in \mathrm{L}_{\mathrm{loc}}$. Then conditions necessary and sufficient in order that, for some $l^{\prime}, f(x) g(x) \sim$ $\mathrm{l}^{\prime} \mathrm{x}^{\mathrm{p}+\mathrm{q}}(\mathrm{C}, \mu)$ whenever $\mathrm{f}(\mathrm{x}) \sim 1 \mathrm{x}^{\mathrm{p}}(\mathrm{C}, \lambda)$ for some $\mathrm{l}$ are that, for some a $(\geqslant 1)$,

(i) $\mathrm{g} \in \mathrm{L}^{\infty}(1, \mathrm{a})$,

(ii) $\frac{1}{\mathrm{u} \cdot \mathrm{a}} \int^{\mathrm{u}} \mathrm{t}^{-\mathrm{q}} \mathrm{g}(\mathrm{t}) \mathrm{dt}=1 \mathrm{o}-\frac{1}{\Gamma(\lambda+1)} \int_{\mathrm{u}}^{\infty}(\mathrm{v}-\mathrm{u})^{\lambda} \mathrm{d} \alpha(\mathrm{v})$ for all $\mathrm{u}>\mathrm{a}$,

where $I_{0}$ is a constant and $\int_{a}^{\infty} t^{\lambda}|d \alpha(t)|<\infty$.

See (1) and (4), where Cesaro summability problems of a similar nature have been considered. section 3 .

Theorem B is deduced from some theorems stated and proved in

\section{Auxiliary Results}

We first define the following subspaces of $\mathrm{L}_{10 \mathrm{c}}$ :

(i) $(\mathrm{C}, \lambda, \mathrm{p}, 1)=\left\{\mathrm{f} / \mathrm{f}(\mathrm{x}) \sim 1 \mathrm{x}^{\mathrm{p}}(\mathrm{C}, \lambda)\right\}$ with the norm defined by

$$
\|f\|=\sup _{t \geqslant 1} t^{-p-\lambda}\left|f_{\lambda}(t)\right| \text {. }
$$

(ii) $\mathrm{C}_{\lambda}=\{\mathrm{f} / \mathrm{f} \in(\mathrm{C}, \lambda, 0,1)$ for some 1$\}$. 
(iii) $\mathrm{N}_{\lambda}^{\mathrm{a}}=\{\mathrm{f} / \mathrm{f} \in(\mathrm{C}, \lambda, 0,0), \mathrm{f}(\mathrm{t})=0$ for $\mathrm{t}<\mathrm{a}, \mathrm{G} \in \mathrm{L}(\mathrm{a}, \infty)\}$, where $G(u)=u f(u)-\int_{1}^{u} f(t) d t$, with $\|f\|=\sup _{t \geqslant a} t^{-\lambda}\left|f_{\lambda}(t)\right|=\sup _{t \geqslant a}\left|\int_{1}^{t} u^{-\lambda-1} G_{\lambda-1}(u) d u\right|$

(iv) $(B, \lambda, p)=\left\{f / f(x)=0\left(x^{p}\right)(C, \lambda)\right\}$

(v) $B_{\lambda}=(B, \lambda, 0)$

(vi) $C_{o}[a, \infty)=\{f / f$ is continuous for $t \geqslant a, f(t) \rightarrow 0$ as $t \rightarrow \infty\}$ with $\|f\|=$ $\sup _{t \geqslant a}|f(t)|$.

$$
\mathrm{BV}[\mathrm{a}, \infty)=\{\mathrm{f} / \mathrm{f} \text { is of bounded variation in }[\mathrm{a}, \infty)\}
$$

The following lemmas will be used in the proofs of the theorems.

LEMMA 1 : If $\left(N_{\lambda}^{a}\right)^{*}$ denotes the dual space of $N_{\lambda}^{a}$, then every continuous linear functional $\Lambda \in\left(N_{\lambda}^{\mathrm{a}}\right)^{*}$ is given by an equation of the form

$$
\begin{gathered}
\Lambda(\mathrm{f})=\frac{1}{\Gamma} \frac{1}{(\lambda)} \int_{\mathrm{a}}^{\infty} \mathrm{f}(\mathrm{u}) \mathrm{du} \int_{\mathrm{u}}^{\infty}(\mathrm{t}-\mathrm{u})^{\lambda-1} \mathrm{~d} \alpha(\mathrm{t}), \text { where } \\
\int_{\mathrm{a},}^{\infty} \mathrm{t}^{\lambda}|\mathrm{d} \alpha(\mathrm{t})|<\infty \text {, the norm of the functional } \Lambda \text { being given by } \\
\|\Lambda\|=\frac{1}{\Gamma(\lambda)} \int_{\mathrm{a}}^{\infty} \mathrm{t}^{\lambda}|\mathrm{d} \alpha(\mathrm{t})| .
\end{gathered}
$$

Proof: Consider the equation $T_{f}(t)=y(t)=t^{-\lambda} \int_{1}^{t}(t-u)^{\lambda-1} f(u) d u \ldots$ Then, $\mathrm{T}_{\mathrm{f}} \in \mathrm{C}_{\mathrm{o}}[\mathrm{a}, \infty)$ whenever $\mathrm{f} \in \mathrm{N}_{\lambda}^{\mathrm{a}}$.

Also, $\left\|\mathrm{T}_{\mathrm{f}_{\mathrm{C}}}\right\|(\mathrm{a}, \infty)=\sup _{\mathrm{t} \geqslant \mathrm{a}}\left|\mathrm{T}_{\mathrm{f}}(\mathrm{t})\right|=\|\dot{\mathrm{f}}\|_{\mathrm{N}_{\lambda}^{\mathrm{a}}}$ and thus the linear operator $\mathrm{T}: \mathrm{N}_{\lambda}^{\mathrm{a}} \rightarrow \mathrm{C}_{\mathrm{o}}[\mathrm{a}, \infty)$ defined by $(2.1)$ is a continuous linear operator which maps $\mathrm{N}_{\lambda}^{\mathrm{a}}$ isometrically onto a subspace of $\mathrm{C}_{\mathrm{O}}[\mathrm{a}, \infty)$. By the Riesz representation theorem, every $\Lambda \bar{\epsilon}\left(\mathrm{C}_{\mathrm{O}}[\mathrm{a}, \infty)\right) *$ is given by

$$
\begin{aligned}
& \Lambda(y)=\int_{a}^{\infty} y(t) d \beta(t), y \in C_{0}[a, \infty) \text {, where } \beta \in B V(a, \infty) \text { and }\|\Lambda\|= \\
& \int_{a}^{\infty}|d \beta(t)| .
\end{aligned}
$$

* Se e (4), Lemma 2. 
Hence, by extending the functional $\Lambda(y)$, we can write every $\Lambda \in\left(N_{\lambda}^{a}\right)^{*}$ in the form

$$
\begin{aligned}
& \Lambda(f)=\int_{a}^{\infty}\left(t^{-\lambda} \int_{1}^{t}(t-u)^{\lambda-1} f(u) d u\right) d \beta(t), \beta \in B V(a, \infty) . \ldots . . . * \\
& \text { i.e. } \Lambda(f)=\int_{a}^{\infty} f(u) d u \int_{u}^{\infty}(t-u)^{\lambda-1} t^{\lambda} d \beta(t) \\
& =\frac{1}{\Gamma(\lambda)} \int_{a}^{\infty} f(u) d u \int_{u}^{\infty}(t-u)^{\lambda-1} d \alpha(t) \text { where } \\
& \alpha(\mathrm{t}) \quad=-\Gamma(\lambda) \int_{\mathrm{t}}^{\infty} \mathrm{u}^{-\lambda} \mathrm{d} \beta(\mathrm{u}) \text { and } \\
& \|\Lambda\|=\int_{a}^{\infty}|\mathrm{d} \beta(\mathrm{t})|=\frac{1}{\Gamma(\lambda)} \int_{a}^{\infty} \mathrm{t}^{\lambda}|\mathrm{d} \alpha(\mathrm{t})| .
\end{aligned}
$$

Since $f \in N_{\lambda}^{a}$, the inversion of the order of integration is justified.

LEMMA 2 : Suppose f.g $\in \mathrm{B}_{\mu}$ for some $\mu$ whenever $\mathrm{f} \in \mathrm{C}_{\lambda}$.

Then, (i) $g \in \mathrm{L}^{\infty}(1, \infty)$,

(ii) There exist $\mathrm{a}(\geqslant 1)$ and $\mathrm{K}$ such that if

$$
\Lambda(f)=\lim _{u \rightarrow \infty} \frac{1}{u} \int_{1}^{u} f(t) g(t) d t \text {, then } \Lambda \in\left(N_{\lambda}^{a}\right)^{*} \text { and }\|\Lambda\| \leqslant K \text {. }
$$

Proof : The necessity of (i) follows trivially since constant functions belong to $\mathrm{C}_{\lambda}$.

Now assume that (ii) is false, i.e. It is false that 'for some a, $K,|\Lambda(f)| \leqslant$ $\mathrm{K}\|\mathrm{f}\|$ whenever $\mathrm{f} \in \mathrm{N}_{\lambda}^{\mathrm{a}}$.

$$
\begin{array}{r}
\text { Now, for } \mu \geqslant 1,-\frac{\mathrm{d}}{\mathrm{du}}\left(\mathrm{u}^{-\mu} \mathrm{I}_{\mu} \mathrm{f}(\mathrm{u}) \mathrm{g}(\mathrm{u})\right)=\mathrm{u}^{-\mu} \mathrm{I}_{\mu-1} \mathrm{f}(\mathrm{u}) \mathrm{g}(\mathrm{u})-\mu \mathrm{u}^{-\mu-1} \mathrm{I}_{\mu} \mathrm{f}(\mathrm{u}) \mathrm{g}(\mathrm{u}) \\
=\mathrm{u}^{-\mu-1} \mathrm{G}_{\mu-1(\mathrm{u})} \\
\ldots \ldots \ldots \ldots
\end{array}
$$

where $G(u)=u f(u) g(u)-f(t) g(t) d t$.

We now define by induction an increasing sequence $\left\{a_{n}\right\}$ tending to $+\infty$ and a sequence of functions $\left\{f_{n}\right\}$ as follows :

Let $a_{O}=1$ and suppose $a_{1}, \ldots . a_{n-1}$ and $f_{1}, \ldots f_{n-1}$ have been defined such that $f_{r} \in N_{\lambda}^{r-1}, r=1, \ldots n-1$. 
Let $G_{r}(u)=u f_{r}(u) g(u)-\int_{1}^{u} f_{r}(t) g(t) d t$ for every $r$.

By (2.2) there exists $f_{n} \in N_{\lambda}^{a_{n-1}}$ such that

$$
\left\|\mathrm{f}_{\mathrm{n}}\right\|<2^{-\mathrm{n}} \text { and } \Lambda\left(\mathrm{f}_{\mathrm{n}}\right)>1
$$

Let $a_{n}=2 a_{n-1}+\sum_{r=1}^{n} \int_{1}^{\infty}\left|G_{r}(u)\right| d u$

Note that $\int_{1}^{\infty}\left|G_{r}(u)\right| d u<\infty$ since $g \in L^{\infty}(1, \infty)$ and $f_{r} \in N_{\lambda}^{a r-1}$.

Now define $f(t)=\sum_{r=1}^{\infty} f_{r}(t)$. Then $f(t)=0$ for $t<1$,

$f \in L_{10 c}$ and $f(t)=\sum_{r=1}^{n} f_{r}(t)$ for $1 \leqslant t \leqslant a_{n}$.

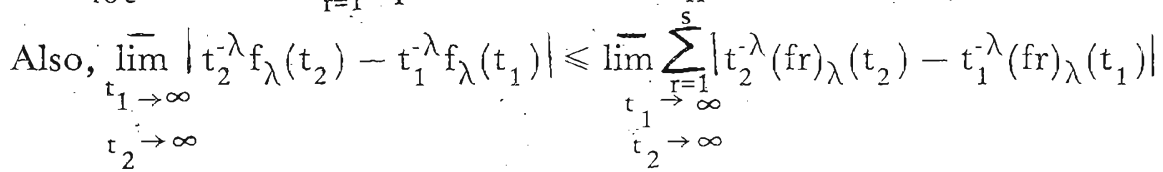

$$
+\varlimsup_{\substack{t_{1} \rightarrow \infty \\ t_{2} \rightarrow \infty}} \sum_{\substack{r=s+1 \\ \infty}}^{\infty} t_{2}^{-\lambda}(f r)_{\lambda}\left(t_{2}\right)-t_{1}^{-\lambda}(f r)_{\lambda}\left(t_{1}\right) \mid
$$

$\leqslant \underset{t_{1} \rightarrow \infty}{ } \sum_{r=s+1}\left\|f_{r}\right\| \leqslant 2 \sum_{r=s+1} 2^{-r}=2^{1-s}$ for arbitary $s \in N$.

$t_{1} \rightarrow \infty$
$t_{2} \rightarrow \infty$

Hence $t^{-\lambda} f_{\lambda}(t) \rightarrow$ a finite limit as $t \rightarrow \infty$, and thus the function $f$ constructed belongs to $\mathrm{C}_{\lambda}$.

$$
\begin{aligned}
& \text { Now, } \int_{n}^{a_{n}} t^{-\mu-1 d t} \int_{1}^{t}(t-u)^{\mu-2} G(u) d u=\sum_{n=1}^{n} \int_{1}^{a_{n}} t^{-\mu-1} d t \int_{1}^{t}(t-u)^{\mu-2} G r(u) d u \\
& =\sum_{r=1}^{n} \int_{n}^{\infty} t^{-\mu-1} d t \int_{1}^{1}(t-u)^{\mu-2} G r(u) d u-\sum_{r=1}^{n} \int_{n}^{2} t_{n}^{-\mu-1} d t \int_{1}^{t}(t-u)^{\mu-2} G r(u) d u \\
& =\sum_{r=1}^{n} \int_{1}^{1} \operatorname{Gr}(u) d u \int_{u}^{\infty}(t-u)^{\mu-2} t^{\mu-1} d t-\sum_{r=1}^{n} \int_{a_{n}}^{\infty} t^{-\mu-1 d t} \int_{1}^{t}(t-u)^{\mu-2} \operatorname{Gr}(u) d u \\
& =\sum_{r=1}^{n} \frac{\Gamma(\mu-1)}{\Gamma(\mu+1)} \Lambda(\mathrm{fr})-\sum_{\mathrm{r}=1}^{\mathrm{n}} \int_{a_{n}}^{\infty} \mathrm{t}^{-\mu-1} \mathrm{dt} \int_{1}^{\mathrm{t}}(\mathrm{t}-\mathrm{u})^{\mu-2} \mathrm{Gr}(\mathrm{u}) \mathrm{du} \\
& \text { But }\left|\sum_{r=1}^{n} \int_{a_{n}}^{\infty} t^{-\mu-1} d t \int_{1}^{t}(t-u)^{\mu-2} \operatorname{Gr}(u) d u\right| \leqslant \sum_{r=1}^{n} \int_{a_{n}}^{\infty} t^{-3} d t \int_{1}^{\infty}|\operatorname{Gr}(u)| d u . \\
& <\frac{1}{a_{n}}-\sum_{r=1}^{n} \int_{1}^{\infty}|\operatorname{Gr}(u)| d u<1 \text { by }(2.5) \text {. }
\end{aligned}
$$

Hence $\int_{1}^{2} t^{\mu \mu-1} d t \int_{1}^{t}(t-u)^{\mu-2} G(u) d u>\frac{\Gamma(\mu-1)}{\Gamma(\mu+1)} n-1$ by (2.4) 
and by (2.3) it follows that $\int_{1}^{a_{n}} \frac{\left(a_{n}-u\right)^{\mu-1}}{a_{n}^{\mu}} f(u) g(u) d u \rightarrow+\infty$ when $\mu>1$. contradicting the fact that $\mathrm{f} . \mathrm{g} \in \mathrm{B}_{\mu}$ for some $\mu$. Hence the necessity of (ii).

LEMMA 3 : If $p>-1, \lambda^{\prime}>\lambda$, then $(C, \lambda, p, 1) \subset\left(C, \lambda^{\prime}, p, 1\right)$ and $(B, \lambda, p)$ $C\left(\mathrm{~B}, \lambda^{\prime}, \mathrm{p}\right)$.

This result is well known. Cf (2), Lemma 3.

LEMMA $4:$ If $p>-1, p+q>-1$ and $g \in(C, \lambda, p, 1)[$ or $(B, \lambda, p)]$, then $\mathrm{h} \in(\mathrm{C}, \lambda, \mathrm{p}+\mathrm{q}, \mathrm{l}) \cdot[$ or $(\mathrm{B}, \lambda, \mathrm{p}+\mathrm{q})]$, where $\mathrm{h}(\mathrm{x})=\mathrm{x}^{\mathrm{q}} \mathrm{g}(\mathrm{x})$.

Cf. (2).Lemma 4.

LEMMA 5 : If $\mathrm{f} \in \mathrm{B}_{\lambda}, \lambda \geqslant 1$, then there exist constants $\mathrm{H}$, $\mathrm{K}$ such that

(i) $\iint_{1}^{t}(t-u)^{\lambda-1}(v-u)^{\alpha} f(u) d u \mid \leqslant H t^{\lambda} v^{\alpha}$ for $v \geqslant t, \alpha \geqslant 0$.

(ii) $\int_{\beta}^{1} \int^{t}(t-u)^{\beta}\left[(v-u)^{\lambda-1}-v^{\lambda-1}\right] f(u) d u \mid \leqslant K t^{\beta+1}\left(t^{\lambda-1}+v^{\lambda-1}\right)$ for $v \geqslant t$,

Proof : The results are trivial for $\lambda=1$, and hence take $\lambda>1$.

(i) Let $\lambda=n+p$ where $n \in I N_{O}, 1<p \leqslant 2$, and $M=\sup _{t \geqslant 1} t^{-\lambda}\left|f_{\lambda}(t)\right|$.

By partial integration we have

$\int_{1}^{\bar{t}} \begin{aligned} & (t-u)^{\lambda-1}(v-u)^{\alpha} f(u) d u=(-1)^{n+1} \int_{1}^{t} f_{n+1}(u)\left(\frac{\partial}{\partial u}-\right)^{n+1}\left[(t-u)^{\lambda-1}\right. \\ & \left.(v-u)^{\alpha}\right] d u\end{aligned}$

$=\sum_{r=0}^{n+1} c_{r} J_{r}$ where $c_{r}$ is independent of $t$ and $v$,

and $J_{r}=\int_{i}^{t}(t-u)^{p+r-2}(v-u)^{\alpha-r} f_{n+1}(u) d u$

$=\frac{(\mathrm{t}-1)^{\mathrm{r}}(\mathrm{v}-1)^{\alpha}}{(\mathrm{v}-1)^{\mathrm{r}}} \int_{1}^{\mathrm{b}_{\mathrm{r}}}(\mathrm{t}-\mathrm{u})^{\mathrm{p}-2} \mathrm{f}_{\mathrm{n}+1}(\mathrm{u}) \mathrm{du}$, where $1 \leqslant \mathrm{~b}_{\mathrm{r}} \leqslant \mathrm{t}$,

by the Second Mean Value Theorem.

By Riesz's Mean Value theorem,

$$
\left|J_{r}\right| \leqslant(t-1)^{r}(v-1)^{\alpha-r} \sup _{1 \leqslant b \leqslant b_{r}^{1}}\left|\int_{r}^{b}(b-u) p-p_{n+1}(u) d u\right|
$$




$$
=(t-1)^{\mathrm{r}}(\mathrm{v}-1)^{\alpha-1} \sup _{1 \leqslant \mathrm{~b} \leqslant \mathrm{~b}_{\mathrm{r}}}\left|\Gamma(\mathrm{p}-1) \mathrm{f}_{\mathrm{n}+\mathrm{p}}^{\prime}(\mathrm{b})\right| \leqslant \Gamma(\mathrm{p}-1) \mathrm{Mt}^{\lambda} \mathrm{v}^{\alpha},
$$

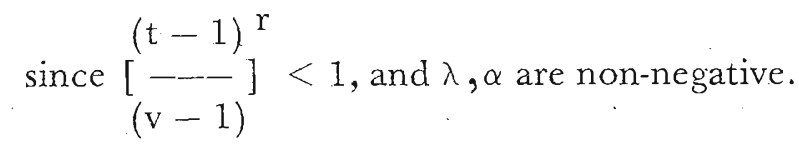

Hence, (2.6) gives (i)

(ii) Take $\beta=\mathrm{n}+\mathrm{p}$ where $\mathrm{n} \in \mathrm{INo}, 0<\mathrm{p} \leqslant 1$. As before, we have

$$
\begin{aligned}
& \int_{1}^{t}(t-u)^{\beta}\left[(v-u)^{\lambda-1}-v^{\lambda-1}\right] f(u) d u \\
&=\left.\left.(-1)^{n+1} \int_{n}^{t} f_{n+1}(u)\left(\frac{\partial}{\partial u}\right)^{n+1}(t-u)^{\beta}[v-u)^{\lambda-1}-v^{\lambda-1}\right]\right\} d u \ldots \ldots(2.7) \\
&\text { But, } \left.\left(\frac{\partial}{\partial u}\right)^{n+1}\left\{(t-u)^{\beta}[v-u)^{\lambda-1}-v^{\lambda-1}\right]\right\}=\operatorname{Co}(t-u)^{\beta-n-1}\left[(v-u)^{\lambda-1}-v^{\lambda-1}\right] \\
& \quad+\sum_{r=1}^{n+1} C_{r}(t-u)^{\beta-n-1+r}(v-u)^{\lambda-1-r} \ldots \ldots . . .
\end{aligned}
$$

As in $(i),\left|\int_{1}^{t}(t-u)^{\beta-n-1+r}(v-u)^{\lambda-1-r} f_{n+1}(u) d u\right| \leqslant \Gamma(\beta-n) M t^{\beta+1} \nu^{\lambda-1}$

$$
\begin{aligned}
\text { Also, } & \left|\int_{1}^{t}(t-u)^{\beta-n-1}\left[(v-u)^{\lambda-1}-v^{\lambda-1}\right] f_{n+1}(u) d u\right| \\
& =\left[v^{\lambda-1}-(v-u)^{\lambda-1}\right]\left|(t-u)^{\beta-n-1} f_{n+1}(u) d u\right| \text { where } 1 \leqslant \eta \leqslant t \\
& \leqslant 2 \Gamma(\beta-n) M t^{\beta+1}\left[t^{\lambda-1}+(\lambda-1) t v^{\lambda-1}\right]
\end{aligned}
$$

$(2.7),(2.8),(2.9)$ and $(2.10)$ give the required result.

\section{Theorems and their Proofs}

THEOREM 1: If f.g $\in \mathrm{B}_{\mu}$ for some uwhenever $\mathrm{f} \in \mathrm{C}_{\lambda}$, then there exists a $(\geqslant 1)$ such that

(i) $g \in \mathrm{L}^{\infty}(1, \mathrm{a})$

(ii) $\frac{1}{\mathrm{u}} \int_{1}^{\mathrm{u}} \mathrm{g}(\mathrm{t}) \mathrm{dt}=1 \mathrm{1}-\frac{1}{\Gamma(\lambda+1)} \int_{\mathrm{u}}^{\infty}(\mathrm{v}-\mathrm{u})^{\lambda} \mathrm{d} \alpha(\mathrm{v})$ for all $\mathrm{u}>\mathrm{a}$,

where $1_{0}$ is a constant, and $\int_{a}^{\infty} v^{\lambda}|d \alpha(v)|<\infty$.

Proof: The necessity of (i) follows from Lemma 2 (i). 
By Lemma 2, there exist. $a_{0}$ and $\mathrm{K}$ such that

$$
\lim _{u \rightarrow \infty}\left|\frac{1}{u_{1}} \int^{u} f(t) g(t) d t\right| \leqslant K\|f\| \text { whenever } f \in N_{\lambda}^{a}
$$

Also, if $f \in N_{\lambda}^{a_{o}}$, then $\frac{I}{u_{1}} \int_{1}^{u}|f(t)| d t \in V\left(a_{o}, \infty\right)$.

Now, (3.1) implies that there exists $a \geqslant a_{0}$ such that if

$$
\Lambda_{u}(f)=\frac{1}{-} \int_{u 1}^{u} f(t) g(t) d t \text {, then, whenever } u>a, \Lambda_{u} \in\left(N_{\lambda}^{a}\right)^{*}
$$

For, if (3.3) is false, by the method used in Lemma 2, we can construct

$$
\left\{b_{n}\right\} \pi, b_{n} \rightarrow+\infty \text { and } f \in N_{\lambda}^{a_{o}} \text { such that } \frac{1}{b_{n}} \int_{1}^{b_{n}} f(t) g(t) d t>n \text {, }
$$

contradicting the fact that $\frac{1}{\mathrm{u}} \int_{1}^{\mathrm{u}} \mathrm{f}(\mathrm{t}) \mathrm{g}(\mathrm{t}) \mathrm{dt}$ is bounded whenever $\mathrm{f} \in \mathrm{N}_{\lambda}^{\mathrm{a}_{\mathrm{o}}}$, which is a consequence of (3.2) and $\mathrm{g} \in \mathrm{L}^{\infty}(1, \infty)$.

Hence, (3.3) and Lemma 1 give : Whenever $\mathrm{u}>\mathrm{a}$,

$$
\begin{aligned}
& \frac{1}{u} \int_{1}^{u} f(t) g(t) d t=\frac{1}{\ulcorner(\lambda)} \int_{a}^{\infty} f(u) d u \int_{u}^{\infty}(t-u)^{\lambda-1} d \alpha(t) \text {, where } \\
& \int_{a}^{\infty} t^{\lambda}|d \alpha(t)|<\infty, \text { for } f \in N_{\lambda}^{a} .
\end{aligned}
$$

Clearly, the function $\mathrm{X}_{(\mathrm{a}, \mathrm{u})}^{\mathrm{a}}$ belongs to $\mathrm{N}_{\lambda}^{\mathrm{a}}$. Hence (3.4) gives

$$
\frac{1}{u} \int_{a}^{u} g(t) d t=\frac{1}{\Gamma(\lambda)} \int_{a}^{u} d t \int_{t}^{\infty}(v-t)^{\lambda-1} d \alpha(v) \text { for all } u>a .
$$

$$
\begin{aligned}
& \text { Since } \int_{\mathbf{a}}^{\infty} \mathrm{t}^{\lambda}|\mathrm{d} \alpha(\mathrm{t})|<\infty, \frac{1}{\Gamma(\lambda)} \int_{\mathbf{a}}^{\mathrm{u}} \mathrm{dt} \int_{\mathrm{t}}^{\infty}(\mathrm{v}-\mathrm{t})^{\lambda-1} \mathrm{~d} \alpha(\mathrm{v}) \\
& =\frac{1}{\Gamma(\lambda)} \int_{a}^{\infty} \mathrm{dt} \int_{\mathrm{t}}^{\infty}(\mathrm{v}-\mathrm{t})^{\lambda-1} \mathrm{~d} \alpha(\mathrm{v})-\frac{1}{\Gamma} \overline{(\lambda)} \int_{\mathrm{u}}^{\infty} \mathrm{dt} \int_{\mathrm{t}}^{\infty}(\mathrm{v}-\mathrm{t})^{\lambda-i} \mathrm{~d} \alpha(\mathrm{v})
\end{aligned}
$$

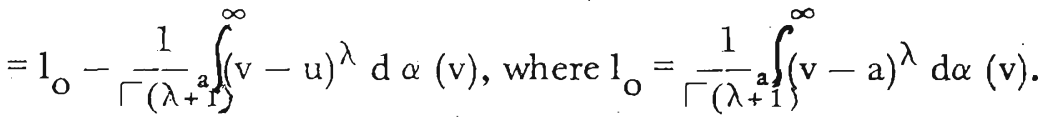

Hence the result, 
THEOREM $2:$ If $\mathrm{f} \in \mathrm{B}_{\lambda}$ and $(\mathrm{i}) \mathrm{h} \in \mathrm{L}^{\infty}(1, \mathrm{a} \mathrm{a})$

(ii) $\quad \frac{1}{u} \int_{a}^{u} h(t) d t=\frac{1}{\Gamma(\lambda+1)} \int_{u}^{\infty}(v-u)^{\lambda} d \alpha(v)$, where $\int_{a}^{\infty} v^{\lambda}|d \alpha(v)|<\infty$, then

$I: \lim _{t \rightarrow \infty} t^{-\lambda} I_{\lambda} f(t) h(t)=-\lim _{t \rightarrow \infty} \frac{t^{-1}}{\Gamma} \int_{(\lambda} \int_{a}^{t} I_{\lambda}\left(v f(\dot{v})-f_{1}(v)\right) d \alpha(v)$.

Proof : Since $f \in B_{\lambda}$, by Lemma 4 we have

$I_{\lambda}\left(v f(v)-f_{1}(v)\right)=o\left(v^{\lambda+1}\right)$, and hence the R.H.S. of I exists, since $\int_{a}^{\infty} v^{i}|d \alpha(v)|<\infty$.

Now (ii) gives $h(u)=-\frac{1}{\Gamma(\lambda+1)} \int_{u}^{\infty}(v-u)^{\lambda} d \alpha(v)-\frac{u}{\Gamma(\lambda)} \int_{u}^{\infty}(v-u)^{\lambda-1} d \alpha(v)$ for $u>a$.

Hence $t^{-\lambda} I_{\lambda} f(t) h(t)=t_{1}^{-\lambda} \int_{\Gamma(\lambda)}^{a}(t-u)^{\lambda-1} f(u) h(u) d u+I_{1}+I_{2}$

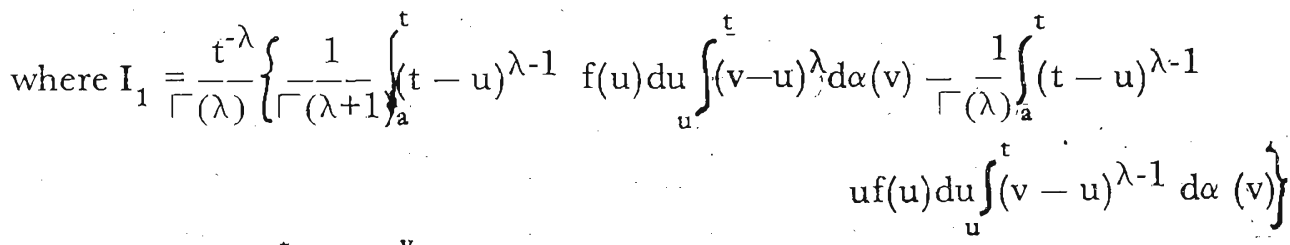

$=\frac{t^{-\lambda}}{\Gamma(\lambda)}\left\{-\frac{1}{\Gamma(\lambda+1)} \int_{a}^{t} d \alpha(v) \int_{a}^{v}(v-u)^{\lambda}(t-u)^{\lambda-1} f(u) d u\right.$

$\left.-\frac{1}{\Gamma(\lambda)} \int_{a}^{t} d \alpha(v) \int_{a}^{v}(v-u)^{\lambda-1}(t-u)^{\lambda-1} u f(u) d u\right\}$

$$
\begin{aligned}
& \text { and } I_{2}=\frac{t^{-\lambda}}{\Gamma(\lambda)}\left\{\frac{1}{\Gamma(\bar{\lambda}+1)} \int_{-a}^{t}(t-u)^{\lambda-1} f(u) d u \int_{\bar{t}}^{\infty}(v-u)^{\lambda} d \alpha(v)\right. \\
& \left.--\frac{1}{\Gamma(\lambda)} \int_{a}^{t}(t-u)^{\lambda-1} \mathrm{f}(\mathrm{u}) \mathrm{du} \int_{\mathrm{t}}^{\infty}(\mathrm{v}-\mathrm{u})^{\lambda-1} \mathrm{~d} \alpha(\mathrm{v})\right\} \\
& =\frac{t^{-\lambda}}{\Gamma(\lambda)}\left\{\left(\Gamma \frac{1}{\Gamma(\lambda+1)}+\frac{1}{\Gamma(\lambda)}\right) \int_{t}^{\infty} d \alpha(v) \int_{a}^{t}(t-u)^{\lambda-1}(v-u)^{\lambda} f(u) d u .\right.
\end{aligned}
$$




$$
\left.-\frac{1}{\Gamma(\lambda)} \int_{t}^{\infty} d \alpha(v) \int_{a}^{t} v(t-u)^{\lambda-1}(v-u)^{\lambda-1} \cdot f(u) d u\right\}
$$

Hence, by Lemma 5(i) we get

$\left|I_{2}\right| \leqslant K_{1} t^{-\lambda} \int_{\mathfrak{i}}^{\infty} t^{\lambda} v^{\lambda}|d \alpha(v)| \rightarrow 0$ as $t \rightarrow \infty$

Now, by (3.6) we gęt $I_{1}-\frac{t^{-1}}{\Gamma(\lambda)} \int_{a}^{t} I_{\lambda}\left(f_{1}(v)-v f(v) d \alpha(v)\right.$

$$
\begin{aligned}
=\frac{\mathrm{t}^{-\lambda}}{\Gamma(\lambda)}\left\{\frac{1}{\Gamma(\lambda+1)} \int_{a}^{\mathrm{t}} \mathrm{d} \alpha(\mathrm{v}) \int_{a}^{\mathrm{v}}(\mathrm{v}-\mathrm{u})^{\lambda}\left[(\mathrm{t}-\mathrm{u})^{\lambda-1}-\mathrm{t}^{\lambda-1}\right] \mathrm{f}(\mathrm{u}) \mathrm{du}\right. \\
\left.-\frac{1}{\Gamma(\lambda)} \int_{a}^{\mathrm{t}} \mathrm{d} \alpha(\mathrm{v}) \int_{\mathrm{a}}^{\mathrm{v}}(\mathrm{v}-\mathrm{u})^{\lambda-1}\left[(\mathrm{t}-\mathrm{u})^{\lambda-1}-\mathrm{t}^{\lambda-1}\right] \mathrm{uf}(\mathrm{u}) \mathrm{du}\right\}
\end{aligned}
$$

Hence $\left|I_{1}-\frac{t^{-1}}{\Gamma(\lambda)} \int_{a}^{t} I_{\lambda}\left(f_{1}(v)-v f(v)\right) d \alpha(v)\right|$

$$
\begin{array}{ll}
\leqslant \mathrm{K}_{2} \mathrm{t}^{-\lambda} \int_{\mathrm{a}}^{\mathrm{t}} \mathrm{v}^{\lambda+1}\left(\mathrm{v}^{\lambda-1}+\mathrm{t}^{\lambda-1}\right)|\mathrm{d} \alpha(\mathrm{v})| & \text { by Lemma } 5 \text { (ii) } \\
\leqslant \mathrm{K}_{2} \mathrm{t}_{\mathrm{a}}^{-\lambda} \int_{1}^{\mathrm{w}} \mathrm{v}^{\lambda+1}\left(\mathrm{v}^{\lambda-1}+\mathrm{t}^{\lambda-1}\right)|\mathrm{d} \alpha(\mathrm{v})|+\underset{\mathrm{w}}{2 \mathrm{~K}_{2} \int^{\infty} \mathrm{v}^{\lambda}|\mathrm{d} \alpha(\mathrm{v})|, \text { where } \mathrm{a}<\mathrm{w}<\mathrm{t} \text {, and }}
\end{array}
$$

Hence $\lim _{w \rightarrow \infty} \lim _{t \rightarrow \infty}\left|I_{1}-\frac{t^{-1}}{\Gamma(\lambda)} \int_{a}^{t} I_{\lambda}\left(f_{1}(v)-v f(v)\right) d \alpha(v)\right|=0$

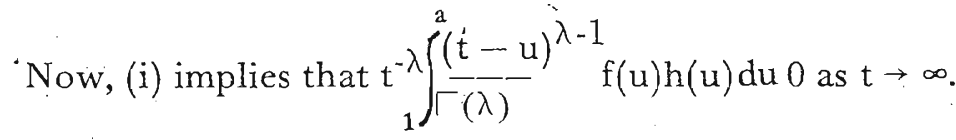

Hence, (3.5), (3.7) and (3.8) give I.

THEOREM 3 : Conditions necessary and sufficient in order that $f . g \in C_{\lambda}$ whenever $f \in C_{\lambda}$ are that, for some a $(\geqslant 1)$

(i) $\mathrm{g} \in \mathrm{L}^{\infty}(1, \mathrm{a})$,

(ii) $\frac{1}{\mathrm{u}} \int_{\mathrm{a}}^{\mathrm{u}} \mathrm{g}(\mathrm{t}) \mathrm{dt}=1_{0}-\frac{1}{\Gamma_{\infty}(\lambda+1)} \int_{\frac{\mathrm{a}}{\mathrm{a}}}^{\infty}(\mathrm{v}-\mathrm{u})^{\lambda} \mathrm{d} \alpha(\mathrm{v})$ for all $\mathrm{u} \geqslant \mathrm{a}$

where $1_{O}$ is a constant and $\int_{a}^{\infty} t^{\lambda}|d \alpha(t)|<\infty$.

Proof : If f.g $\in \mathrm{C}_{\lambda}$ whenever $\mathrm{f} \in \mathrm{C}_{\lambda}$, then $\mathrm{f}$.g $\in \mathrm{B}_{\lambda}$ whenever $\mathrm{f} \in \mathrm{C}_{\lambda}$, and by Theorem 1, (i) and (ii) are necessary. 
If (i) and (ii) hold, then $g(u)=1_{o}+h(u)$, where $h(u)$ is as in Theorem 2.

Hence $t^{-\lambda} I_{\lambda} f(t) g(t)=1_{0} t^{-\lambda} f_{\lambda}(t)+t^{-\lambda} I_{\lambda} f(t) h(t) \rightarrow$ a finite limit as $t \rightarrow \infty$ whenever $f \in C_{\lambda}$, by Theorem 2 .

THEOREM 4 : If $\dot{\mu} \geqslant \lambda \geqslant 1, \mathrm{p}+\mathrm{q}>-1, \mathrm{p}>-1$ then conditions necessary and sufficient in order that, for some l',

f.g $\in\left(\mathrm{C}, \mu, \mathrm{p}+\mathrm{q}, \mathrm{l}^{\prime}\right)$ whenever $\mathrm{f} \in(\mathrm{C}, \lambda, \mathrm{p}, 1)$ for some 1 are that for some $a(\geqslant 1)$,

(i) $\mathrm{g} \in \mathrm{L}^{\infty}(1, \mathrm{a})$

(ii) $\frac{1}{-} \int_{\mathrm{u}}^{\mathrm{u}} \mathrm{t}^{-\mathrm{q}} \mathrm{g}(\mathrm{t}) \mathrm{dt}=1 \mathrm{o}-\frac{1}{\Gamma(\lambda+1)} \int_{\mathrm{u}}^{\infty}(\mathrm{v}-\mathrm{u})^{\lambda} \mathrm{d} \alpha(\mathrm{v})$ for all $\mathrm{u}>\mathrm{a}$,

where $1_{0}$ is constant, $\int_{a}^{\infty} t^{\lambda}|d \alpha(t)|<\infty$.

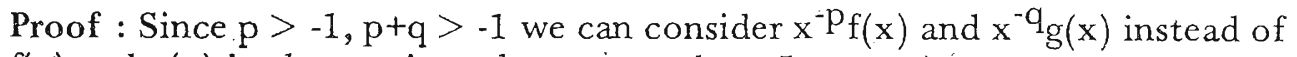
$\mathrm{f}(\mathrm{x})$ and $\mathrm{g}(\mathrm{x})$ in the previous theorem, and use Lemma 4 .

Hence Theorem 1 gives the necessity of (i) and (ii).

Again by Theorem 3, (i), (ii) and $f \in(C, \lambda, p, 1)$ imply that f.g $\in\left(\mathrm{C}, \lambda, \mathrm{p}+\mathrm{q}, \mathrm{l}^{\prime}\right)$ for some l', and hence f.g $\in\left(\mathrm{C}, \mu, \mathrm{p}+\mathrm{q}, \mathrm{l}^{\prime}\right)$, by Lemma 3 .

This completes the proof.

\section{References:}

1. BORWEIN, D. (1954) J. Lond. Math. Soc. 29: 198- 206.

2. BORWEIN, D. (1956) J. Lond. Math. Soc. $25: 289-302$.

3. BOSANQUET, L. S. (1954) Mathematika; $1: 24-44$.

4. SARGENT, W. L.C. (1952) J. Lond. Math. Soc. $27: 401-413$.

5. YOGACHANDRAN, C: (1981) J. Natn. Sci. Coun. Sri Lanka $9: 17-23$. 